\title{
ANALYSIS OF CRACK EVOLUTION IN CONCRETE THROUGH COMBINED ACOUSTIC EMISSION MONITORING AND MESOSCALE MODELLING
}

\author{
J. SALIBA* ${ }^{*}$, M. MATALLAH ${ }^{\dagger}$, A. LOUKILI ${ }^{\dagger \dagger}$, J.P. REGOIN ${ }^{\dagger \dagger}$, D. GRÉGOIRE $^{\dagger \dagger \dagger}$, L. \\ VERDON $^{\dagger \dagger \dagger}$, G. PIJAUDIER-CABOT ${ }^{\dagger \dagger}$, M. SBARTAI ${ }^{*}$, S. MOREL ${ }^{*}$ \\ * Université de Bordeaux, Institut de Mécanique et d'Ingénierie (I2M), Département Génie Civil et \\ Environnemental (GCE), Talence, France j.saliba@i2m.u-bordeaux1.fr \\ ${ }^{\dagger}$ RISAM Université de Tlemcen, BP 230, Algeria \\ ${ }^{\dagger \dagger}$ LUNAM Universite, UMR-CNRS 6183, Ecole Centrale de Nantes, Institut de Recherche en Génie Civil et \\ Mécanique, (GeM), Nantes, France \\ ${ }^{\dagger \dagger}$ Université de Pau et Pays Adour, Laboratoire des Fluides Complexes et leurs Réservoirs, LFC-R \\ UMR5150, Anglet, France
}

Key words: Concrete, acoustic emission, mesoscopic modelling, damage

\begin{abstract}
In this paper, the fracture process zone (FPZ) is investigated on unnotched and notched beams with different notch depths. Three-point bending tests have been realized on plain concrete under crack mouth opening displacement (CMOD) control. Crack growth is monitored by applying the acoustic emission (AE) technique. The comparison with a numerical model is also realized by using a mesoscopic approach. Such an approach is of particular interest in the analysis of interactions between the cementitious matrix and aggregates. Several AE parameters are examined during the entire loading process, and show that the relative notch depth influences the AE characteristics, the process of crack propagation, and the brittleness of concrete. The numerical load-CMOD curves show that the mesoscopic modelling reproduces well the notch effect and concrete failure. In order to improve our understanding of the FPZ, the width and length of the FPZ are followed based on the AE source locations maps in parallel with the numerical damage fields. An important energy dissipation is observed at the crack initiation in unnotched beams.
\end{abstract}

\section{INTRODUCTION}

Fracture of concrete is accompanied by the formation and evolution of an inelastic zone, referred to as the fracture process zone (FPZ), around the propagating crack tip. The existence of the FPZ is responsible of the nonlinear behaviour of concrete, and leads to complex phenomena like size effects. In fact, the length and the width of the FPZ are strongly influenced by the sizes of specimens/structures. Many researchers tried to characterize the FPZ and its evolution during crack extension in order to obtain size independent fracture parameters for the application of fracture 
mechanics of concrete.

The objective of this paper is to characterize the FPZ and its evolution during the fracture process in unnotched and notched concrete beams with different notch depths based on the $\mathrm{AE}$ technique and finite elements calculations. The AE technique allows us a continuous realtime data acquisition, and thus the damage evolution during loading tests can be recorded. It is a passive method that has been proved to be very effective to locate microcracks and to study different failure modes in concrete structures $[1,2,3,4]$. It presents a remarkable potential of applications, and has been used in the past to examine the influence of different parameters on the FPZ, such as the effect of aggregates [5, 6], porosity [7], creep [8, 9], notch depth [10], specimen geometry and type of loading [11]. The damage is then evaluated based on either statistical analysis of $\mathrm{AE}$ activity [12] or quantitative and signal-based techniques [4].

Several numerical models were capable to capture important characteristics that emerge in failure process and $\mathrm{AE}$ measurements $[13,14$, 15]. A thorough investigation is here performed on this subject by modelling the behaviour of concrete at the mesoscopic scale. The mesoscale modelling presents many advantages in the understanding of the fracture process (i.e. the transition from diffuse damage to localized damage and final discrete failure) and the effect of concrete heterogeneities (size, volume fraction, shape of aggregates...) and mechanical characteristics of the components on macroscopic properties and the fracture behavior of concrete $[16,17,18]$. In particular, the multi-scales approach was found to be very useful to study the size effect on the strength of plain concrete structures $[19,20,21]$. In the present paper, the damage model used is the isotropic damage model developed by Fichant $\&$ al. [22], which is implemented in the finite element code Cast $3 \mathrm{~m}$.

In section 2, experimental methods and damage model are presented. Then, fracture measurements are analysed, and the characterization of crack evolution at different loading stages is examined through $\mathrm{AE}$ technique and mesoscopic modelling. Finally, the effects of both the notch to depth ratio and size effect on fracture growth are described based on the experimental and numerical observations.

\section{EXPERIMENTAL PROCEDURE}

\subsection{Materials and specimens}

The experiments were realized by Grégoire $\&$ al. and reported in [23]. In the present work, the comparison between notched and unnotched beams is realized. The tests realized on notched beams were conducted on beams with depth of $200 \mathrm{~mm}$, length of $700 \mathrm{~mm}$, and effective span equal to $500 \mathrm{~mm}$. Two notch to depth ratio values of 0.2 and 0.5 were considered and labeled SN200 and LN200, respectively. Tests realized on unnotched beams were conducted on beams with the same sizes as earlier (labeled UN200) and on beams with depth of $100 \mathrm{~mm}$, length of $350 \mathrm{~mm}$ and effective span equal to $250 \mathrm{~mm}$ (labeled UN100). The thickness was kept constant for all the beams and equal to $50 \mathrm{~mm}$.

Tests were conducted under closed-loop crack mouth opening displacement (CMOD) control. The CMOD measurement consists in recording the distance between two alumina plates glued on the bottom surface of the beam, on each side of the initial notch. For unnotched beams, the alumina plates were glued at a distance from midspan equal to half the depth of the beam because the location of the fracture process zone initiating from the surface was indeterminate (details may be found in [23]).

\subsection{Acoustic emission technique}

The AE system comprises an eight channel AEwin system, a general-purpose interface bus (PCI-DISP4) and a PC for data storage analysis. A 2D analysis with an AEwin algorithm is performed for the localization of AE events. In this study, 4 piezoelectric sensors with a frequency of $50-200 \mathrm{kHz}$ and a resonance frequency of $150 \mathrm{kHz}$ are used. The transducers are placed around the expected location of the FPZ to minimize errors in the AE event localization. They are placed on the specimens with silicon grease as the coupling agent. The 
sensors form a rectangular grid location on one side of $\left(75 \times 120 \mathrm{~mm}^{2}\right)$ for UN200 beams, $(60$ $\left.\mathrm{x} 120 \mathrm{~mm}^{2}\right)$ for UN100 beams, $\left(105 \mathrm{x} 120 \mathrm{~mm}^{2}\right)$ for SN200 beams and $\left(110 \times 120 \mathrm{~mm}^{2}\right)$ for LN200 beams [15].

The detected signals are amplified with a 40 $\mathrm{dB}$ gain differential amplifier. The recorded $\mathrm{AE}$ amplitudes range from 0 to $100 \mathrm{~dB}$. In order to overcome the background noise, the signal detection threshold is set at a value of $35 \mathrm{~dB}$ slightly above the background noise. The acquisition system is calibrated before each test using a pencil lead break procedure HSUNIELSEN [24]. Location accuracy is measured in the range of $5 \mathrm{~mm}$ by applying the pencil lead fracture at a known location of the specimen. The measured effective velocity is equal to $3800 \mathrm{~m} / \mathrm{s}$. Each waveform is digitized and stored, and signal descriptors such as rise time, absolute energy, duration, amplitude, average frequency and counts to peak are captured by AEwin system. The absolute energy is defined as the integral of the squared voltage signal divided by the reference resistance (10K-Ohm) over the duration of $\mathrm{AE}$ waveform. This feature is the true energy measure of the AE hit, and the unit is expressed in atto-joules (aJ).

\section{DAMAGE MODEL}

The chosen damage model is the isotropic model developed by Fichant et al. [22]. It allows to represent the unilateral effect due to crack closure effect (a decomposition of the stress tensor into a positive and negative part is introduced) and to obtain objective results independent of the length of the finite elements by controlling the fracture energy. This model is a simplified version of the microplan model [25], and is based on the relationship between the total stress and the effective stress $\underline{\underline{\sigma}}(\underline{y})$ of the material:

$$
\underline{\underline{\sigma}}(\underline{y})=C_{\sim}^{0}(\underline{y}): C_{\sim}^{-1}(\underline{y}, \underline{\underline{\varepsilon}}(\underline{y})): \underline{\underline{\sigma}}(\underline{y})
$$

where $\underset{\sim}{C}(\underline{y})$ is the initial stiffness of the material phases (the material is considered isotropic and linear elastic), $C^{-1}(\underline{y}, \underline{\underline{\varepsilon}}(\underline{y}))$ the stiffness of the damaged material, $\underline{\underline{\sigma}}(\underline{y})$ the local stress field in each point $\underline{y}$ and $\underline{\underline{\varepsilon}}(\underline{y})$ the local strain field. For the isotropic version of the model, the expression linking the effective stress and the total stress is :

$$
\underline{\underline{\sigma}}=(1-d) \underline{\underline{\tilde{\sigma}}}
$$

where $d$ is the scalar value of the isotropic damage that only depends on the equivalent strain computed according to the elastic strain tensor $\underline{\underline{\varepsilon}}^{e}$ :

$$
\varepsilon_{e q}=\sqrt{\underline{\underline{\varepsilon}}^{e}: \underline{\underline{\varepsilon}}^{e}}
$$

Damage increases when the equivalent strain $\varepsilon_{e q}$ is higher than the threshold strain $\varepsilon_{d 0}$ with $\varepsilon_{d 0}=\frac{f_{t}}{E}$ where $\mathrm{E}$ is the Young modulus and $f_{t}$ tensile strength. The evolution of the damage variable, due to external mechanical loads, has an exponential form:

$$
d=1-\frac{\varepsilon_{d 0}}{\varepsilon_{e q}} \exp \left[B_{t}\left(\varepsilon_{d 0}-\varepsilon_{e q}\right)\right]
$$

where $B_{t}$ represents a damage parameter to control the slope of the strain softening constitutive relationships and is calculated as a function of the width $h$ of the finite element, and the fracture energy $G_{f}$.

$$
\frac{G_{f}}{h}=\frac{f_{t} \varepsilon_{d 0}}{2}+\frac{f_{t}}{B_{t}}
$$

The Digital Concrete Model was developed with the aim to have a 'realistic' representation of cement-based materials by taking into account the random size distribution of heterogeneities [26]. A specific algorithm was developed to make a spatial and random distribution of these phases on the basis of a finite element grid. First of all, all grid elements have mortar properties. Then, aggregates are placed in the grid from the biggest to the smallest, according to the aggregate size distribution, such that no overlapping is obtained with other particles already placed. The properties of the grid elements located in 
an aggregate area are those of aggregates. A regular grid is considered for the mesoscopic mesh with an 8 node quadrilateral finite element

\subsection{Application on concrete at the mesoscale}

The considered mesoscale for modelling the behaviour of concrete is the scale at which the material can be observed as a set of coarse aggregates embedded in a mortar matrix. Here, coarse aggregates are inclusions of size greater than $5 \mathrm{~mm}$ following the experimental aggregate size distribution, whereas mortar matrix is a mixture of finer aggregates and the cement paste (Table 1). The aggregates volume represents $30 \%$ of the total volume of concrete. The stability of results depending on the size of the specimen and the maximum diameter of the inclusion was performed in [26], and the criteria are taken into consideration here with specimen sizes equal to four times the highest inclusion diameter (L/Dmax>4) and finite element size equal to 0.8 time the smallest inclusion diameter. Note that the random aggregates distribution has a non-negligible effect on the stress concentration that can influence the cracking process and the path of the macrocrack [9].

Table 1: Aggregate size distribution of concrete

$\begin{array}{cccccc}\phi(\mathrm{mm}) & 5 & 6 & 6.35 & 8 & 10 \\ V_{f}(\%) & 4.8 & 3 & 4.2 & 7.8 & 10.2\end{array}$

A regular mesoscopic mesh related to the middle part of concrete beams is generated, and two homogeneous concrete blocks are attached to the left and right end of the beam with progressively larger mesh to avoid stress concentration. The finite element meshes of concrete beams are presented in Figure 1. The loading is applied as an incremental vertical displacement of a rigid plate fixed at the top middle of the beam. The behavior of the plate is assumed linear elastic with a Young's modulus ten times greater than that of concrete.

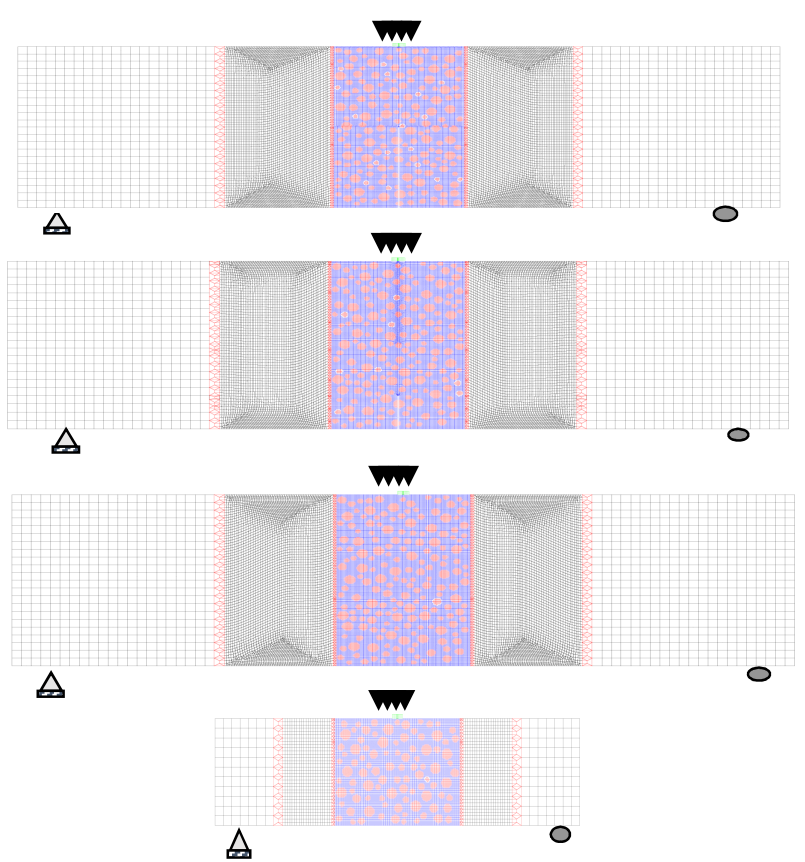

Figure 1: Meso-macro meshing and boundary conditions of concrete beams under three pointbending test

The numerical simulations are conducted under plane stress condition. The mechanical characteristics of aggregates are obtained from the literature: Young's modulus of $60 \mathrm{GPa}$ and tensile strength of $6 \mathrm{MPa}$ are considered.

Numerical simulations are then performed to validate the choice of the mechanical properties defined for mortar and aggregates. For the homogeneous part, the parameters are determined by the mechanical concrete properties. The parameters used at the mesoscopic scale are summarized in Table 2.

Table 2: Model parameters for mortar and aggregates

\begin{tabular}{ccccc}
\hline \multicolumn{5}{c}{ Elastic and failure parameters } \\
\hline & $\begin{array}{c}v_{0} \\
(\mathrm{GPa})\end{array}$ & $f_{t}$ & $G_{f}$ \\
& 26 & 0.2 & 3.8 & 60 \\
\hline Mortar & 60 & 0.2 & 6 & 80 \\
\hline Aggregate & & & &
\end{tabular}




\section{STRUCTURAL RESPONSE ON THREE-POINT BENDING TEST}

The influence of size and notch effect on the mechanical properties is investigated by comparing experimental and numerical loadCMOD curves. Calculations are performed with the digital concrete model for three mesh generations representing three different random aggregates distributions in the volume. The mesoscopic modelling reproduces well the experimental load-CMOD curves (Figure 2). Note that the nominal strength and the postpeak region of the load-displacement curves slightly vary with the random distribution of the aggregates. In addition, the results show a more important maximal stress for unnotched beams in comparison with the notched ones, with effects of both the notch depth and the specimen size.

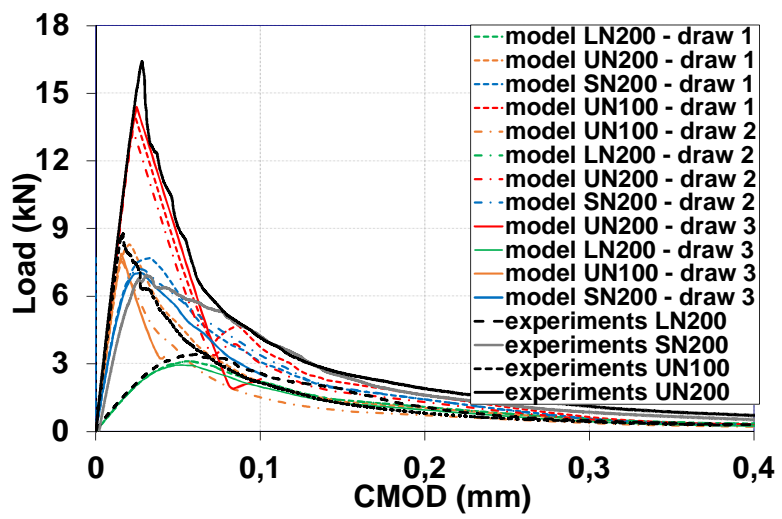

Figure 2: Comparison of numerical and experimental load versus CMOD curves for UN200, UN100, SN200 and LN200 beams.

In order to have a better understanding of the notch and size effects, the three point bending tests have been monitored with the $\mathrm{AE}$ technique, and numerical simulations have been realized.

\subsection{Effect of relative notch depth on $\mathrm{AE}$}

\section{characteristics and numerical damage}

\section{evolution}

Damage quantification is computed based on the AE activity represented by the cumulative number of AE hits (Figure 3a). The same processing is applied to the mesoscopic modelling by considering that each microcrack corresponds to a damaged Gauss point of the finite elements (i.e. when the damage parameter $d$ is greater than 0 ). The cumulated number of damaged finite elements is plotted in Figure $3 \mathrm{~b}$. For the beams with depth of $200 \mathrm{~mm}$, the results show that, as the notch depth decreases, the total number of $\mathrm{AE}$ hits increases requiring higher energy for overcoming interfacial bond along the ligament length.

For UN100, the rate of $\mathrm{AE}$ hits is similar to that for UN200 beams at the beginning, and reaches later values between the total number of AE hits for LN200 (presenting the same ligament length) and the total number of $\mathrm{AE}$ hits for UN200, which could be related to the additional energy required for the crack initiation in unnotched beams and to different crack lengths. The numerical damage evolution shows the same trend with most important damaged finite elements at the crack initiation site for unnotched beams and a total number of damaged finite elements proportional to the ligament length, and thus a good proportionality between the AE activity and the damage evolution observed through the numerical model. The cumulated AE energy and the strain energy released by finite elements follow the same trend, and give us information on initiation of cracks that progress up to failure, and thus can be related to the fracture energy measured on a global scale. Note that, even though the mesoscopic modelling can represent the stochastic properties of concrete, however the cumulative AE hits and damaged finite elements are only qualitatively comparable because the numerical concrete microstructure is not totally consistent with that of the original concrete. 

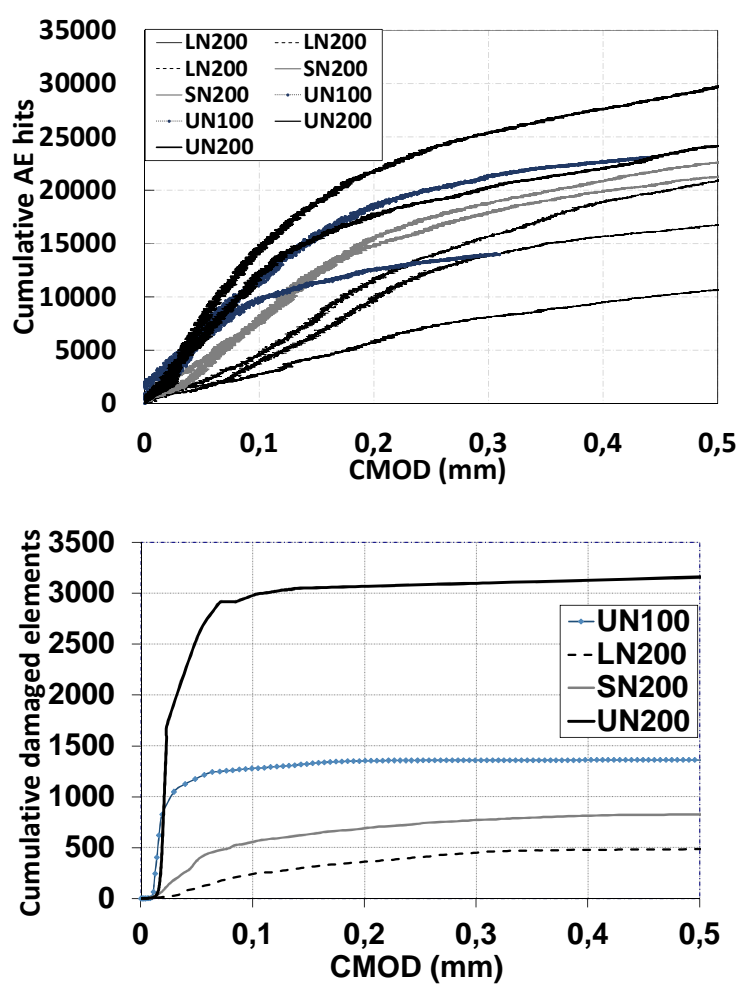

Figure 3: a) cumulative AE hits and b) cumulative damaged gauss point in function of CMOD for UN200, UN100, SN200 and LN200 beams.

\subsection{AE damage localization and numerical damage fields}

During the formation of a crack, energy is emitted as an elastic wave and propagates from the crack location to the AE transducers at the specimen surface. The localization map of $\mathrm{AE}$ events is based on arrival times of the first wave at each transducer and their respective velocity in the concrete specimen. Once the arrival time is picked, the least-square method is used to estimate the event location. The cumulative acoustic events are placed in 2D. The detected $\mathrm{AE}$ events of notched and unnotched beams are presented over a window covering the specimen height, with a width of $300 \mathrm{~mm}$ centered with respect to the notch (Figure 4). Each plotted point indicates a detected AE source.

In addition to the number of $\mathrm{AE}$ events, the parameters of the $\mathrm{AE}$ signal can provide valuable information on the fracture process. The initiation and propagation of cracks in concrete are generally correlated to AE signals amplitude. Extensive studies have shown that the absolute acoustic energy is also a significant parameter to characterize an event. Thus, the 2D localization maps of $\mathrm{AE}$ events are classified in terms of their levels of energy. Six energy levels are defined. It can be seen that $\mathrm{AE}$ events of higher energy levels are located in the core of the FPZ [9] outlining the crack path. The digital concrete model permits also to simulate the cracking process by following numerical damage fields. The crack opening is calculated with the numerical method suggested by Matallah et al. [27].

The localization maps of AE sources, the numerical damage localization and the crack opening are plotted at $50 \%$ of the maximum strength in the post-peak region (Figure 4). The crack opening images have been plotted on deformed beams to provide more visibility.

At the peak load, few AE events are detected, and damage begins to develop in a distributed manner. The localization maps show that the AE events appear first for LN200 beams, then for SN200, UN100 and UN200, respectively. The damage fields are localized in front of the notch at the mortar-aggregates interface at the beginning. Then, they increase with loading to develop in the matrix, and localize in a single macro-crack.

At $50 \%$ in the post peak region, the localization maps also show different crack evolution with a more remarkable crack length for notched beams. The damage fields and crack opening obtained from the mesoscopic modelling also show also different cracking evolutions. Cracks begin to join up together, and propagate in the mortar matrix exhibiting different realistic features as crack bridging and branching.

For unnotched beams, the localization of $\mathrm{AE}$ events begins earlier for UN100 than for UN200, with a more significant crack length, also indicating a more ductile behaviour for small beams due to different stress gradient along the ligament length. Further, a crack branching has been observed in the localization maps of UN200 and SN200 which could be due to an aggregate interlock, and indicating a rough and complex fracture surface along the ligament length of the beams.

During the terminal post peak region, the number of $\mathrm{AE}$ events continue to increase with 
a lower rate due to the stable propagation of the crack. AE events are generated as a consequence of different toughening mechanisms through the crack faces on a micro and meso level, such as bridging and friction.

In order to improve our understanding of the FPZ, the width and length of the FPZ have been followed based on the AE source locations maps in parallel with the numerical investigation.

SN200
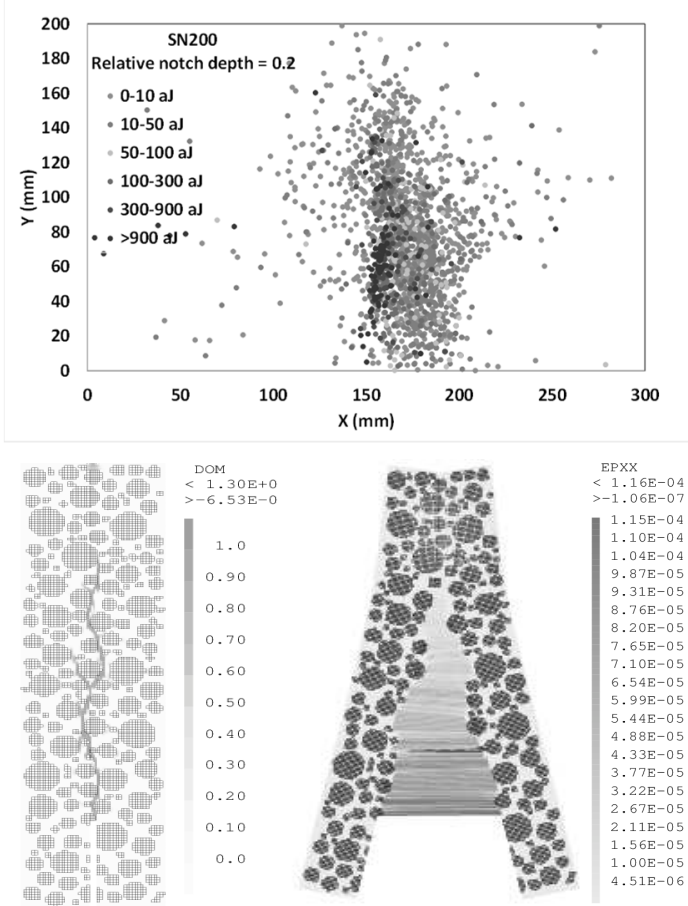

\section{LN200}
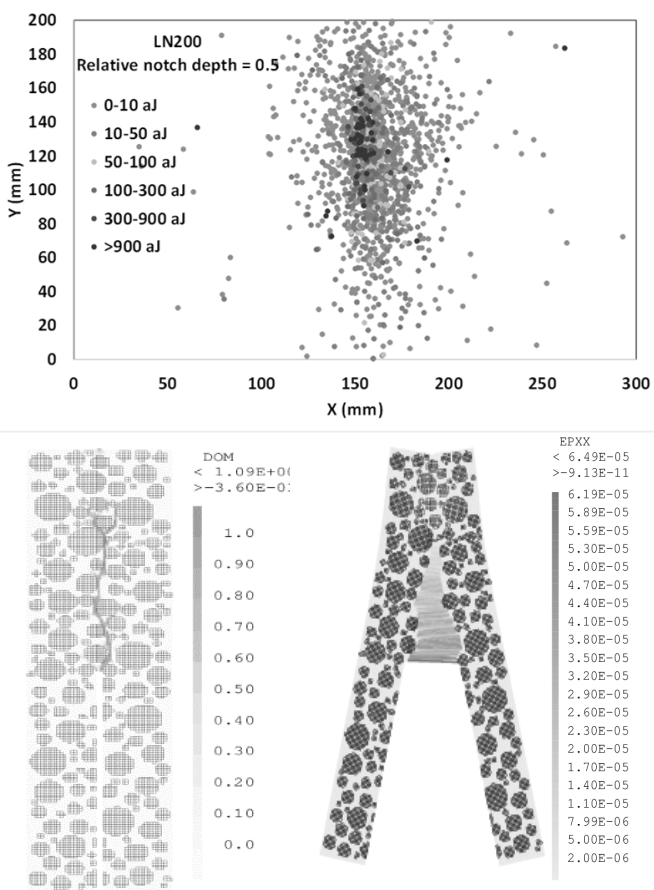

\section{UN200}
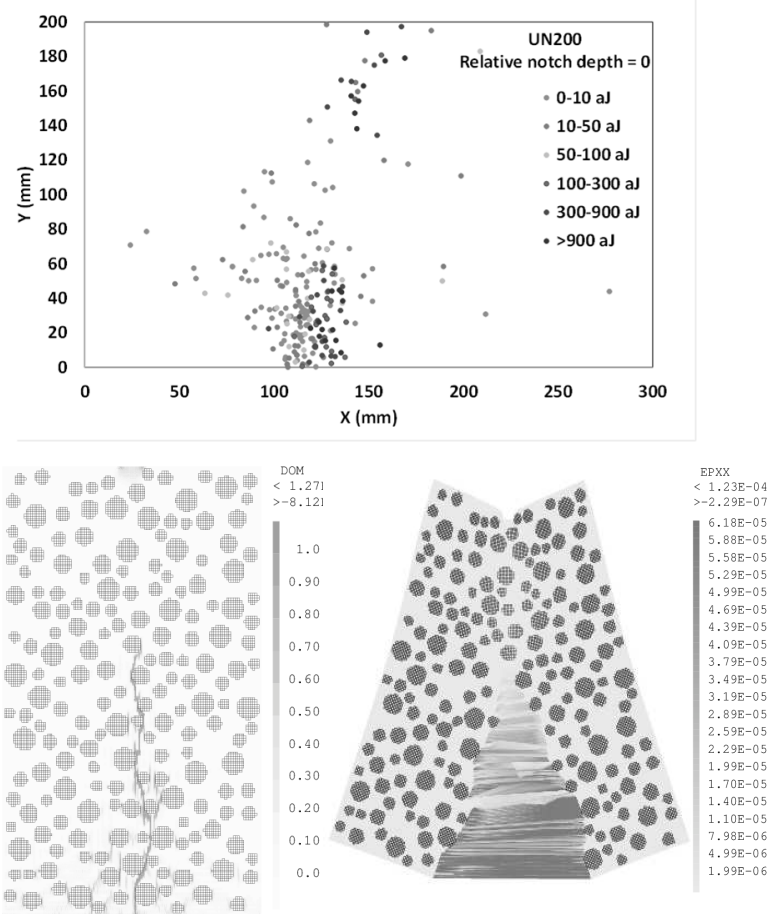

\section{UN100}
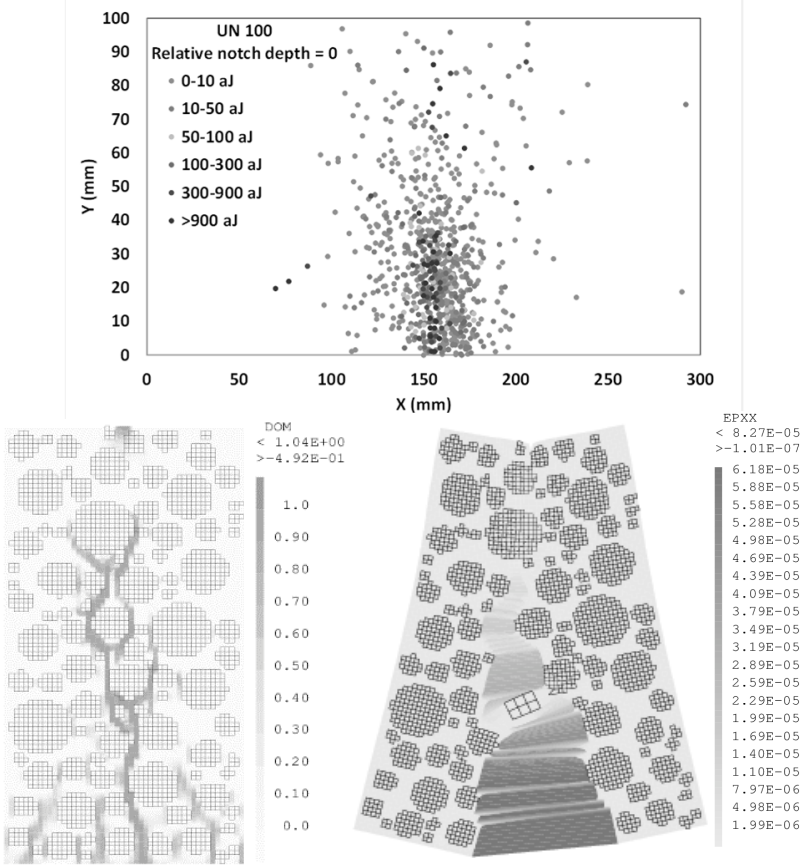

Figure 4: Localization maps of the AE events, numerical damage fields and crack openings at $50 \%$ of the maximal strength in the post-peak region for SN200 (A), LN200 (B), UN200 (C) and UN100 (D)beams. 


\subsection{Characterization of the FPZ}

Different approaches were proposed to determine the length and width of the damaged zone using probability, statistic laws and different empirical relations [12].

In our study, the length of the FPZ is estimated based on AE events density along the ligament length. Figure 5(a) shows the density of $\mathrm{AE}$ events at each Y location, at the end of the rupture test for UN200, UN100, SN200 and LN200 beams. The crack propagates form the front boundary toward the back one induced by free surface. For notched beams, it can be seen that, ahead of the notch tip, the number of AE events progressively increases due to the front boundary, attains the maximum value (Nmax), then remains almost constant for some distance along the ligament length due to the steady state propagation of the crack, and decreases as the crack propagates towards the back boundary. The curves clearly follow the energy dissipation trend shown by the boundary effect model (which allow to model size effect on the specific fracture energy) and can be used to compute the real fracture energy. The dissipated energy during crack propagation from the front boundary to the back one can be taken into account by assuming a trilinear variation of the local fracture energy over the ligament length [29, 30]. However, for unnotched beams, the $\mathrm{AE}$ events density presents a remarkable peak near the front boundary indicating a more significant energy dissipation for crack initiation. Then it decreases along the ligament length.

The occurrence of $\mathrm{AE}$ events can be considered as a criterion to be adopted in order to follow the crack propagation through the beam depth for different loading levels. Therefore, the length of the FPZ can be defined as the length of the segment from the notch tip to the intersection of the histogram with the horizontal line located at $20 \%$ of Nmax. Fracture examination in concrete through combined techniques as digital image correlation and X-rays can give additional information. However, the monitoring of the evolution of the fracture length with $\mathrm{AE}$ technique shows similar trends as those above mentioned, and proved to be quantitatively acceptable $[1,31]$. The relative fracture length, i.e. the ratio of fracture length from the crack mouth to the ligament length of the beam, obtained from the AE technique at different loading intervals, is plotted in Figure 5 (b) for UN200, UN100, SN200 and LN200 beams. .

Fracture initiates earlier at $90 \%$ of the maximum strength in the pre-peak regime for the LN200 beams (followed by SN200), while the fracture growth of unnotched beams starts after the peak load. The relative fracture length in LN200 beam is more significant in comparison with those related to SN200, UN100 and UN200, and the relative fracture length of UN100 has values between those of UN200 and SN200 beams. The fracture growth is relatively abrupt at the beginning, and decreases at the end. A similar behaviour was also observed while studying size effect with a more remarkable crack length for smaller beams [31].
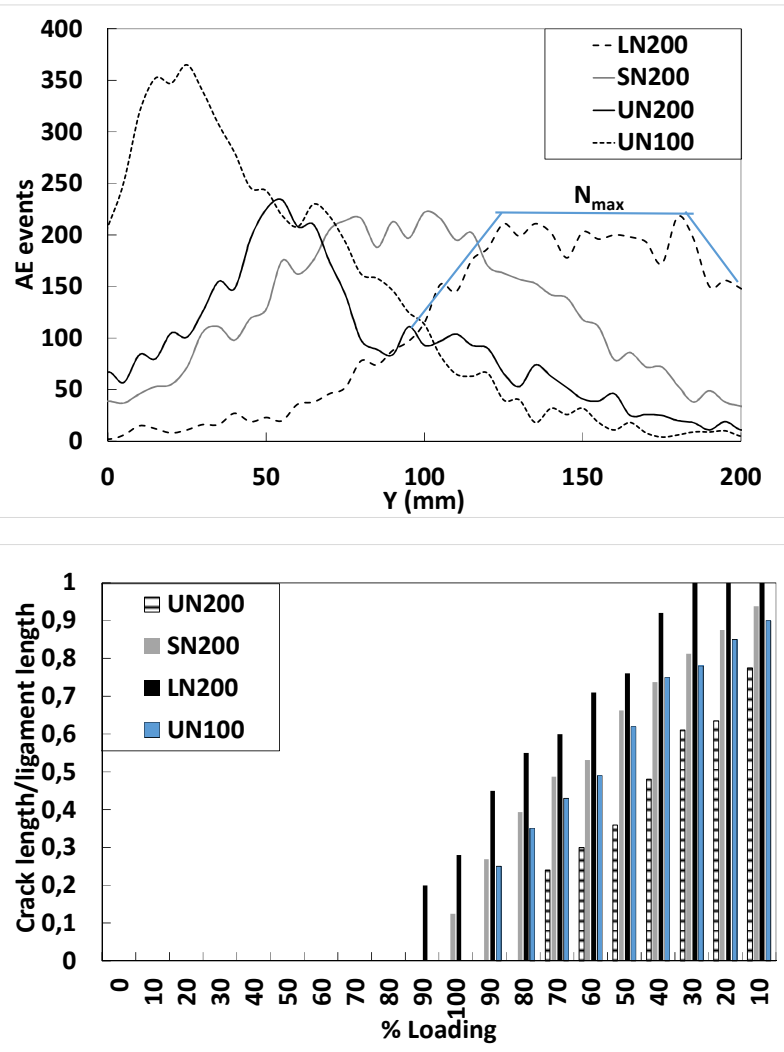

Figure 5: a) Cumulative AE events at each Y location at the end of the rupture test and b) evolution of relative crack length with load steps for UN200, UN100, SN200 and LN200 beams 
The FPZ length was also computed using the mesoscopic approach by studying the evolution of the tangential stress $\sigma_{\theta \theta}$. This latter was numerically obtained along the crack path determined according to the post processing method developed by Matallah \& al. [26]. Since the maximum value of the stress perpendicular to the crack path is obtained at the crack tip, the length of the FPZ was considered as the distance between the stress free crack tip ( $\left.\sigma_{\theta \theta}=0\right)$ and the crack tip $\left(\sigma_{\theta \theta}=f_{t}\right)$ [32] (Figure 6). The results show that the crack appears at $40 \%$ and $50 \%$ of the maximum strength for notched beams with the mesoscopic modelling. However the same trends are observed as those related to the $\mathrm{AE}$ analysis with delayed cracks for unnotched beams at $90 \%$ and $100 \%$ of the maximum strength for UN100 and UN200, respectively. In fact, a large number of $\mathrm{AE}$ hits have been registered at the beginning with the $\mathrm{AE}$ technique without being localized due to the attenuation of emitted weak amplitude hits (for the source located in 2D, a wave must reach at least three sensors). In addition, the $\mathrm{AE}$ analysis does not give us precise information about the tip of the stress free crack because the criteria used for determining the length of the FPZ may cause a loss of information since we cannot exactly localize the crack tip position. All those elements may be responsible of the observed discrepancy.

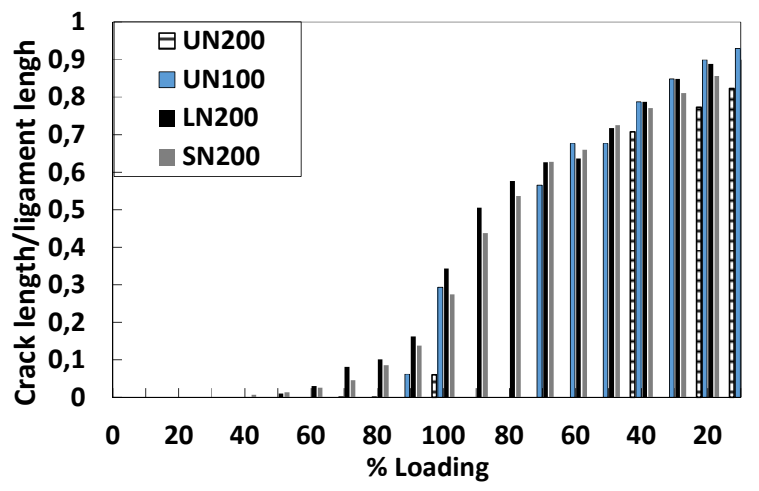

Figure 6: Evolution of relative crack length with load steps for UN200, UN100, SN200 and LN200 beams based on the mesoscopic modelling.
Based on the localization maps, the width of the FPZ has also been estimated based on the horizontal line located at $20 \%$ of Nmax which separate the zone with a high number of events representing the localization of microcracking and the outside zone where the level of damage is lower [12] (Figure 7). The discrepancy between the widths of the FPZ is relatively important, and this could be due to different sources of error: the localization of AE events is realized in 2D on one face of the specimen. However, the crack path inside the thickness of the beams is different (in 3D) due to the internal heterogeneity of the material. In addition, the crack path is dominated by the distribution of aggregates and other softening mechanisms, and the width of the FPZ cannot correctly be determined when the main crack branches into microcracks in multiple directions. However, the results show that the width of FPZ tends to increase with the decrease of the relative notch depth. This behaviour was also observed in Otsuka and al. [1]

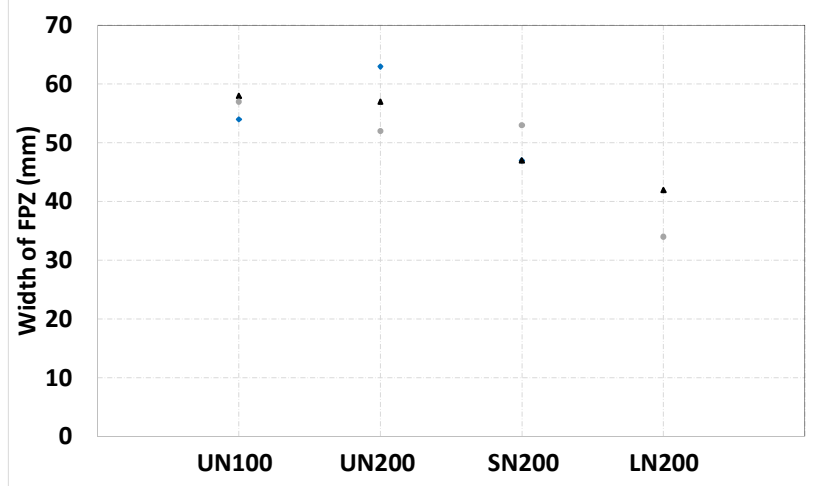

Figure 7: a) Cumulative AE events at each X location at the end of the rupture test and $b$ ) the width of the FPZ for UN200, UN100, SN200 and LN200 beams

Those differences in length and width of the FPZ show a non-uniform stress distribution in the cross-sections of the beams with different sizes and different values of the relative notch depth which could be responsible, in combination with the softening behaviour of concrete, of the size effect in concrete structures. 


\section{CONCLUSION}

An experimental and numerical study is presented in order to monitor fracture growth during three-point bending tests in notched and unnotched concrete beams. The mesoscale modelling reproduces well the notch effect on the load deflection curves of beams under bending. The total number of AE hits increases with the decrease of the relative notch depth, and shows a linear proportionality with the evolution of the damaged Gauss points obtained from the numerical model. The fracture process zone length has also been monitored with $\mathrm{AE}$ technique and the numerical model. The evolution of the fracture length is highly dependent on the relative notch depth due to the stress gradient. The initiation of the fracture is detected earlier in notched beams compared to unnotched beams with a higher relative crack length respectively. An additional energy dissipation for crack initiation has also been observed in unnotched beams. Additional studies are needed using a 3D mesoscopic modelling and a 3D localization algorithm for the $\mathrm{AE}$ analysis in order to evaluate the crack profile in $3 \mathrm{D}$.

\section{ACKNOWLEDGMENTS}

The MCIA (Mésoscentre de Calcul Intensif Aquitain) where all simulations were performed is gratefully acknowledged. The National Research Agency is thanked for the financial support and all the partners of ENDE project.

\section{REFERENCES}

[1] Otsuka, K., Date, H. 2000. Fracture process zone in concrete tension specimen. Eng. Fract. Mech. 65:111131.

[2] Landis, E.N., Shah, S.P. 1995. The influence of microcracking on the mechanical behaviour of cement based materials. Cem. Based Mat. 2:105-118.

[3] Chen, B., Liu, J. 2004. Experimental study on AE characteristics of threepoint-bending concrete beams. Cem. \&
Concr. Res. 34:391-397.

[4] Grosse, C.U., Fink, F. 2006. Quantitative evaluation of fracture processes in concrete using signalbased acoustic emission techniques. Cem. Concr. Comp. 28:330-336.

[5] Wu, K., Chen, B., Yao, W. 2001. Study of the influence of aggregate size distribution on mechanical properties of concrete by acoustic emission technique. Cem. Concr. Res. 31:919923.

[6] Chen, B., Liu, J. 2004. Effect of aggregate on the fracture behaviour of high strength concrete. Constr. Build. Mater. 18:585-590.

[7] Haidar, K., Pijaudier-Cabot, G., Dubé, J.F., Loukili, A. 2005. Correlation between the internal length, the fracture process zone and size effect in model materials. Mater. \& Struct. 38:201-210.

[8] Saliba, J., Loukili, A., Grondin, F., Regoin, J.-P. 2012. Influence of basic creep on cracking of concrete shown by the Acoustic Emission technique. Mater. \& Struct. 45:1389-1401.

[9] Saliba, J., Loukili, A., Grondin, F., Regoin, J.-P. 2014. Identification of damage mechanisms in concrete under high level creep by the acoustic emission technique. Mater. \& struct. 47:1041-1053

[10] Zhang, D., Wu, K. 1999. Fracture process zone of notched threepoint bending concrete beams. Cem. \& Concr. Res. 29:1887-1892.

[11] Labuz, J.F., Cattaneo, S., Chen, L. 2001. Acoustic emission at failure in quasi-brittle materials. Constr. build. Mater. 15:225-233.

[12] Hadjab, H.S., Thimus, J.F., Chabaat, M. 2007. The use of acoustic emission to investigate fracture process zone in notched concrete beams. Curr. Sci. 93:648-653.

[13] Vesely V., Frantik P. 2010. Reconstruction of a fracture process zone during tensile failure of quasibrittle materials. Appl. and comput. Mech. 4:237-250. 
[14] Zhu W.C., Zhao X.D., Kang Y.M., Hei C.H., Tian J. 2010. Numerical simulation on the acoustic emission activities of concrete. Mater. Struct. 43:633-650

[15] Grégoire, D., Verdon, L., Lefort, V., Grassl, P., Saliba, J., Regoin, J.P., Loukili, A., Pijaudier-Cabot, G. 2015. Mesoscale analysis of failure in quasibrittle materials : comparison between lattice model and acoustic emission data. Inter. J. Numer. Anal. Meth. Geomech. DOI 10.1002.nag.2363.

[16] van Mier J.G.M., Van Vliet M.R.A. 2003. Influence of microstructure of concrete on size/scale effects in tensile fracture. Eng. Fract. Mech. 70:2281-2306.

[17] Leite J.P.B., Slowik V., Mihashi H. 2004. Computer simulation of fracture processes of concrete using mesolevel models of lattice structures. Cem. Concr. Res. 34:1025-1033.

[18] Kozicki J., Tejchman J. 2007. Effect of aggregate structure on fracture process in concrete using 2D lattice model. 59(4-5):365-384.

[19] Grassl, P., Grégoire, D., Solano, L.R., Pijaudier-Cabot G. 2012. Mesoscale modelling of the size effect on the fracture process zone of concrete. Int. J. Solids Struct. 49:1818-1827.

[20] Saliba J., Grondin F., Loukili A. Morel S. 2014. Numerical investigation of the size effects on the creep damage coupling. Procedia Mater. Sci.. 3:10381043.

[21] Ince R., Arslan A., Karihalo B.L. 2003. Lattice modelling of size effect in concrete strength. Eng. Fract. Mech.. 70:2307-2320.

[22] Fichant, S., Pijaudier-Cabot, G., La Borderie, C. 1997. Contiuum damage modelling: approximation of crack induced anisotropy. Mech. Res. Commun. 24 (2) :109-114.

[23] Grégoire, D., Rojas-Solano, L.B., Pijaudier-Cabot, G. 2013. Failure and size effect for notched and unnotched concrete beams. Int. $J$.
Numer. Anal. Meth. Geomech. 37:14341452.

[24] RILEM TC212-ACD recommendation. 2010. Acoustic emission and related NDE techniques for crack detection and damage evaluation in concrete. Mater. Struct. 43:1177-1181.

[25] Carol I., Bazant Z.P. 1997. Damage and plasticity in microplane theory. Int. J. Solids Struct. 34(29):3807-3835.

[26] Grondin, F., Dumontet, H., Ben Hamida, A. Mounajed, G., Boussa, H. 2007. Multi-scales modelling for the behaviour of damaged concrete. Cem. Concr. Res. 37 :1453-1462.

[27] Matallah, M., La Borderie, C., Maurel, O. 2010. A practical method to estimate crack openings in concrete structures. Int. J. Numer. Anal. Meth. 34:1615-1633.

[28] Grassl P., Bazant Z.P. 2009. Random lattice-particle simulation of statistical size effect in quasi-brittle structures failing at crack initiation. $J$. Eng. Mech. 135:85-92.

[29] Duan, K, Hu, X, Wittmann, F.H. 2007. Size effect on specific fracture energy of concrete. Eng. Fract. Mech. 74:87-96.

[30] Muralidhara, S., Raghu Prasad, B.K., Karihaloo, B.L., Singh, R.K. 2011. Size-independent fracture energy in plain concrete beams using tri-linear model. Constr. build. Mater. 25:30513058.

[31] Alam, S.Y., Saliba, J., Loukili, 2014. A. Fracture examination in concrete through combined digital image correlation and acoustic emission techniques. Constr. build. Mater. 69:232-242.

[32] Aissaoui N., Matallah M., Loukili A. 2014. Size Effect in Concrete Structures A mesoscopic approach Vs Fracture Mechanics. 32èmes Rencontres Universitaires de Génie Civil, AUGC, Polytech Orléans, 4-6 june. 